\title{
BMJ Open Age differences in clinical features and outcomes in patients with COVID-19, Jiangsu, China: a retrospective, multicentre cohort study
}

\section{Huanyuan Luo, ${ }^{1}$ Songqiao Liu, ${ }^{2}$ Yuancheng Wang, ${ }^{3}$ Penelope A Phillips-Howard, ${ }^{1}$} Shenghong Ju (D) , ${ }^{3}$ Yi Yang, ${ }^{2}$ Duolao Wang (D) ${ }^{1}$

To cite: Luo H, Liu S, Wang Y, et al. Age differences in clinical features and outcomes in patients with COVID-19, Jiangsu, China: a retrospective, multicentre cohort study. BMJ Open 2020;10:e039887. doi:10.1136/ bmjopen-2020-039887

\section{- Prepublication history and} additional material for this paper are available online. To view these files, please visit the journal online (http://dx.doi. org/10.1136/bmjopen-2020039887).

$\mathrm{HL}$ and SL contributed equally.

Received 28 April 2020 Revised 29 August 2020 Accepted 04 September 2020

Check for updates

(C) Author(s) (or their employer(s)) 2020. Re-use permitted under CC BY-NC. No commercial re-use. See rights and permissions. Published by BMJ.

For numbered affiliations see end of article.

Correspondence to Professor Yi Yang; yiyiyang2004@163.com and Professor Duolao Wang; Duolao.Wang@Istmed.ac.uk

\section{ABSTRACT}

Objectives To determine the age-specific clinical presentations and incidence of adverse outcomes among patients with COVID-19 in Jiangsu, China.

Design and setting Retrospective, multicentre cohort study performed at 24 hospitals in Jiangsu, China. Participants 625 patients with COVID-19 enrolled between 10 January and 15 March 2020.

Results Of the 625 patients (median age, 46 years; 329 (52.6\%) men), 37 (5.9\%) were children (18 years or younger), 261 (41.8\%) young adults (19-44 years), $248(39.7 \%)$ middle-aged adults (45-64 years) and 79 (12.6\%) elderly adults (65 years or older). The incidence of hypertension, coronary heart disease, chronic obstructive pulmonary disease and diabetes comorbidities increased with age (trend test, $p<0.0001, p=0.0003, p<0.0001$ and $p<0.0001$, respectively). Fever, cough and shortness of breath occurred more commonly among older patients, especially the elderly, compared with children $\left(\chi^{2}\right.$ test, $p=0.0008,0.0146$ and 0.0282 , respectively). The quadrant score and pulmonary opacity score increased with age (trend test, both $p<0.0001$ ). Older patients had many significantly different laboratory parameters from younger patients. Elderly patients had the highest proportion of severe or critically-ill cases $\left(33.0 \%, \chi^{2}\right.$ test $\left.p<0.0001\right)$, intensive care unit use $\left(35.4 \%, \chi^{2}\right.$ test $\left.p<0.0001\right)$, respiratory failure $\left(31.6 \%, \chi^{2}\right.$ test $\left.p<0.0001\right)$ and the longest hospital stay (median 21 days, Kruskal-Wallis test $\mathrm{p}<0.0001$ ).

Conclusions Elderly ( $\geq 65$ years) patients with COVID-19 had the highest risk of severe or critical illness, intensive care use, respiratory failure and the longest hospital stay, which may be due partly to their having a higher incidence of comorbidities and poor immune responses to COVID-19.

\section{INTRODUCTION}

COVID-19 infection causes a wide spectrum of disease, which may lead to respiratory failure and organ failure, leading to death. Most of the available studies prior to the COVID-19 pandemic found that people of all ages are susceptible to SARS-CoV-2 infection but noted higher positivity rates in real-time reverse transcriptase-PCR (RT-PCR) assays

\section{Strengths and limitations of this study}

- This cohort consists of almost all COVID-19 patients in Jiangsu province, with a population of over 80 million. Findings are, therefore, representative of the hospitalised patient population in the whole province. They are inclusive across the range of disease severity, so are subject to less selection bias.

- The study includes imported and local cases with different types of exposure.

- This is an observational exploratory study and our results may thus be subject to possible confounding factors and false-positive error.

and hospitalisation burden in older people. ${ }^{1-3}$ Similar to SARS, deaths and adverse clinical outcomes have been found to be more common in the elderly with known comorbidities for patients with COVID-19. ${ }^{4-6}$ Evidence suggests that asymptomatic carriers were more common among middle-aged people in close contact with infected family members. ${ }^{7}$ One study found that elderly patients with COVID-19 had some different clinical features compared with younger patients. ${ }^{8}$ Because age is a host factor that leads to a higher risk of severe COVID-19 and worse prognosis, it is important to better understand age-related susceptibility and pathology. However, published data on age differences in clinical features and clinical outcomes associated with COVID-19 remain scarce. This study aimed to investigate differences in clinical characteristics, disease severity and clinical outcome burden in different age groups of patients with COVID-19.

\section{METHODS}

Study design and participants

This retrospective cohort study included all patients who met the study's inclusion and 
exclusion criteria. The inclusion criterion was up to 15 March 2020, all patients diagnosed with COVID-19 in Jiangsu province according to the 'Diagnosis and Treatment Protocol for Novel Coronavirus Pneumonia (Trial Version 7)' released by the National Health Commission and National Administration of Traditional Chinese Medicine of China. ${ }^{9}$ The only exclusion criterion was any patient with no available medical records. A standard criterion was set for discharge: a patient's body temperature must be normal for more than 3 days, symptoms were resolved (if there were symptoms) and RT-PCR assays (throat swab samples, with at least 1 day sampling interval) showed two consecutive negative results.

\section{Data collection and definition of variables}

Epidemiological, clinical, laboratory and radiologic parameters on admission; disease severity (asymptomatic, mild, moderate, severe and critically ill) and clinical outcomes data were extracted from medical records. A clustered onset was defined as the occurrence of two or more confirmed COVID-19 cases in the same cluster/ group within 14 days, such as family, community, hospital, working place or public place. A clustered onset could occur from interpersonal transmission via close contact with, or joint exposure to, a confirmed COVID-19 case. Other cases not meeting the conditions of a clustered onset were classified as 'single onset'. Disease severity was assessed at days 1, 2, 3, 4, 5, 6, 7 and 14 after admission, except for those who were discharged, and the highest degree of disease severity during the 14-day follow-up was selected for analysis. Data on mortality and other clinical outcomes were available until 15 March 2020. Asymptomatic infection was defined as the absence of clinical symptoms but with a positive nucleic acid test result. Mild disease was defined as having mild clinical symptoms and the absence of imaging manifestations of pneumonia in CT scans. Moderate disease was defined as the presence of fever, respiratory tract symptoms or other symptoms, and imaging manifestations of pneumonia. Severe disease was defined as the presence of at least one of the following conditions: respiratory distress, respiratory rate $\geq 30$ breaths/min; oxygen saturation in resting state $\left(\mathrm{SpO}_{2}\right) \leq 93 \%$; or arterial partial pressure of oxygen $\left(\mathrm{PaO}_{2}\right) /$ fraction of inspired oxygen $\leq 300 \mathrm{~mm} \mathrm{Hg}(1 \mathrm{~mm}$ $\mathrm{Hg}=0.133 \mathrm{kPa}$ ). Critically ill was defined as having respiratory failure requiring mechanical ventilation, shock or combined organ failure requiring intensive care unit (ICU) monitoring and treatment. We categorised the population into four age groups: children (18 years or younger), young adults (19-44 years), middle-aged adults (45-64 years) and elderly adults (65 years or older).

All of the patients in Jiangsu had a high-resolution CT thorax examination to identify lung lesions. CT images were assessed visually by two radiologists who had more than 5 years of working experience in chest imaging. The radiologists were blinded to the patients' characteristics. Quadrant scores were the sum of the number of quadrants containing pulmonary opacities extending from the proximal to the distal end of the chest, with a score ranging from 0 to 4 . For pulmonary opacity, bilateral lungs were scored manually and assigned an estimated percentage of pulmonary opacity relative to the whole lung, rounded to the nearest $5 \%$.

\section{Statistical analysis}

Normally distributed variables were summarised using mean (SD) by group and compared using analysis of variance tests, while skewed distributed variables were summarised using median (IQR) by group and compared using Kruskal-Wallis tests. Categorical variables were summarised using frequency and percentage and compared using $\chi^{2} /$ Fisher exact test. To assess the linear trend effect of age on demographic and clinical variables, generalised linear models were employed with age (in years) as the only predictor. Normal distribution and identity link function were used for continuous variables, whereas binomial distribution and logit link function were used for binary variables. Analyses were performed using SAS V.9.4 (SAS Institute) and a two-sided p $<0.05$ was considered statistically significant.

\section{Patient and public involvement}

Patients and the public were not involved in the design, conduct, reporting, or dissemination plans of this research.

\section{RESULTS}

Of the 721 suspected cases with possible COVID-19 during the study period, 631 patients were found to be RT-PCR positive for COVID-19. Only 625 patients were included in the study with complete data (figure 1). The median age was 46 years (IQR, 32-57; range, $0.75-96$ years) and $329(52.6 \%)$ were men (table 1$)$. Thirty-seven $(5.9 \%)$ were children, 261 (41.8\%) young adults of 19-44 years, $248(39.7 \%)$ middle-aged of $45-64$ years and $79(12.6 \%)$ elderly of 65 years or older.

There was no significant difference in the proportion of men and women in each age group (table 1). The disease onset was clustered among a significantly higher proportion of young patients aged 18 years and below compared with older patients (aged 18 years and younger, young adult, middle-aged and elderly patients: $78.4 \%, 43.3 \%, 52.8 \%$ and $53.2 \%$, respectively, $\mathrm{p}=0.0005)$. The comorbidities incidence of hypertension, coronary heart disease, chronic obstructive pulmonary disease and diabetes increased with age (trend test, $\mathrm{p}<0.0001$, $\mathrm{p}=0.0003, \mathrm{p}<0.0001$ and $\mathrm{p}<0.0001$, respectively). Fever, cough and shortness of breath occurred significantly more commonly among young adult, middle-aged and elderly patients compared with children $(67.8 \%, 68.5 \%$, $65.8 \%$ vs $35.1 \%$, $\mathrm{p}=0.0008 ; 57.9 \%, 56 \%, 54.4 \%$ vs $29.7 \%$, $\mathrm{p}=0.0146 ; 1.9 \%, 3.2 \%, 8.9 \%$ vs $0 \%, \mathrm{p}=0.0282$, respectively). The frequencies of these symptoms were similar among different age groups of adults except for shortness of breath which was dramatically more common $(8.9 \%)$ 

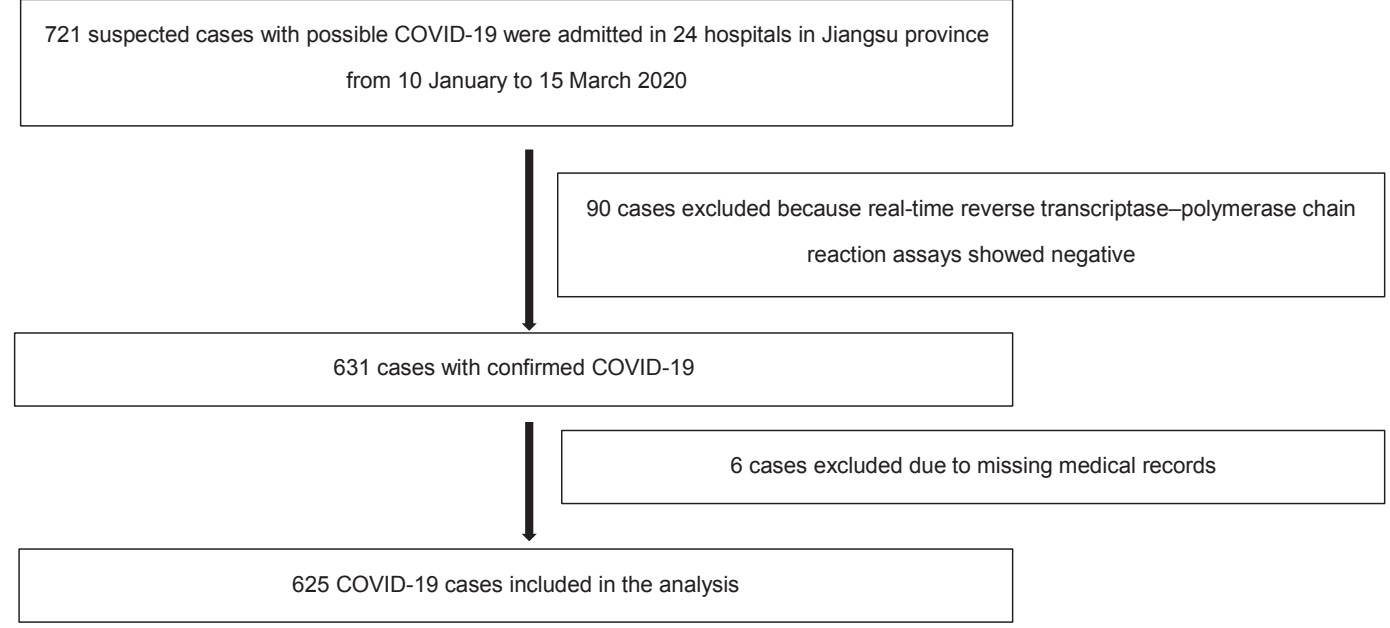

Figure 1: Study flow diagram

Figure 1 Study flow diagram.

in elderly adult patients aged 65 years or older. Compared with children and young adult patients, the incidence of smoking and drinking alcohol was significantly (twice the rate) higher among middle-aged and elderly patients. Three vital sign parameters on admission: systolic blood pressure (SBP) ( $\mathrm{mm} \mathrm{Hg}$ ), diastolic blood pressure (DBP) $(\mathrm{mm} \mathrm{Hg})$ and $\mathrm{SpO}_{2}(\%)$, changed linearly with age (trend tests, all $\mathrm{p}<0.0001$ ). The quadrant score and pulmonary opacity score increased with age (trend tests, both $\mathrm{p}<0.0001)$.

Significant differences were observed in some laboratory test results (table 2). Older patients tended to have lower $\mathrm{PaO}_{2}(\mathrm{~mm} \mathrm{Hg})$ and partial pressure of carbon dioxide $(\mathrm{mm} \mathrm{Hg})$ in blood gas analysis. In blood tests, they had lower white blood cell count $\left(10^{9} / \mathrm{L}\right)$, lymphocyte count $\left(10^{9} / \mathrm{L}\right)$, haemoglobin level $(\mathrm{g} / \mathrm{L})$ and platelet count $\left(10^{9} / \mathrm{L}\right)$. In organ function tests, these patients had higher levels of alanine aminotransferase $(\mathrm{U} / \mathrm{L})$ and creatinine $(\mu \mathrm{mol} / \mathrm{L})$ and lower albumin levels $(\mathrm{g} / \mathrm{L})$. In inflammatory factor tests they had higher levels of C-reactive protein $(\mathrm{mg} / \mathrm{L})$; and in coagulation function tests shorter activated partial thromboplastin time (s) and higher levels of fibrinogen $(\mathrm{g} / \mathrm{L})$ and D-dimer $(\mathrm{mg} / \mathrm{L})$.

The proportion of patients who received supportive treatment and antiviral and antibiotic therapy increased significantly with patients' age, except for the very rare procedure of continuous renal replacement therapy and extracorporeal membrane oxygenation treatment and the very common use of interferon among different age groups (online supplemental table S1).

The proportion of patients with severe or critical illness was 33\% among elderly patients, compared with $13 \%$ among middle-aged patients, $2.3 \%$ among young adult patients and $0 \%$ among children $(\mathrm{p}<0.0001)$ (table 3$)$.
By the end of the study, none of the patients had died and all 625 patients had been discharged. The ICU rate (trend test, $\mathrm{p}<0.0001$ ), respiratory failure rate (trend test, $\mathrm{p}<0.0001$ ) and length of hospital stays (trend test, $\mathrm{p}<0.0001$ ) increased with age (table 4$)$. The proportion of patients requiring ICU care $(\mathrm{p}<0.0001)$ and developing respiratory failure $(\mathrm{p}<0.0001)$ among elderly patients was $35.4 \%$ and $31.6 \%$, respectively, compared with $14.5 \%$ and $12.5 \%$ among middle-aged patients, $2.3 \%$ and $1.9 \%$ among young adult patients and none among children, respectively. Elderly patients also had longer hospital stays (median (IQR), 21.0 (14.0-26.0) days) than all other age groups (15.0 (11.0-21.0) for children, 14.0 (11.0-19.0) for young adults and 17.0 (13.0-22.0) for middle-aged adults, $\mathrm{p}<0.0001$ ).

\section{DISCUSSION}

To our knowledge, this is the largest cohort of 625 patients followed to date, to assess age differences in clinical features and clinical outcomes associated with COVID19 . We observed that the majority $(\sim 80 \%)$ of COVID-19 cases were among young adult patients of 19-44 years and middle-aged patients of 45-64 years, with the remainder of patients comprising children of 18 years or under $(5.9 \%)$ and elderly patients of 65 years or over $(12.6 \%)$. This is consistent with a previous study from Korea which reported $6.3 \%$ of cases with COVID-19 were children under 19 years old, a study which had tested the broadest and hence the most representative population during this study period. ${ }^{10}$

Our study showed that compared with adults, children were more likely to get infected through cluster 


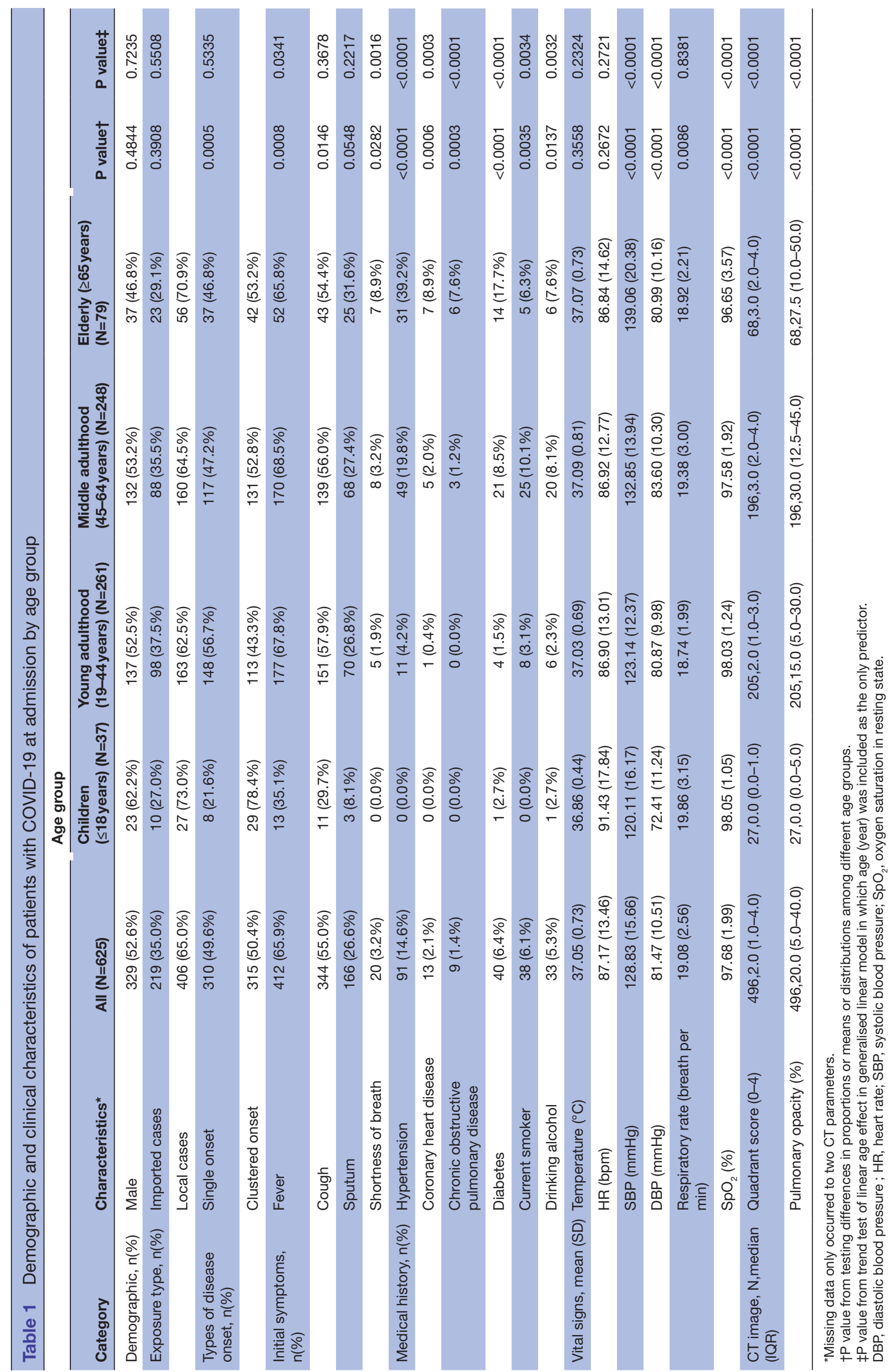




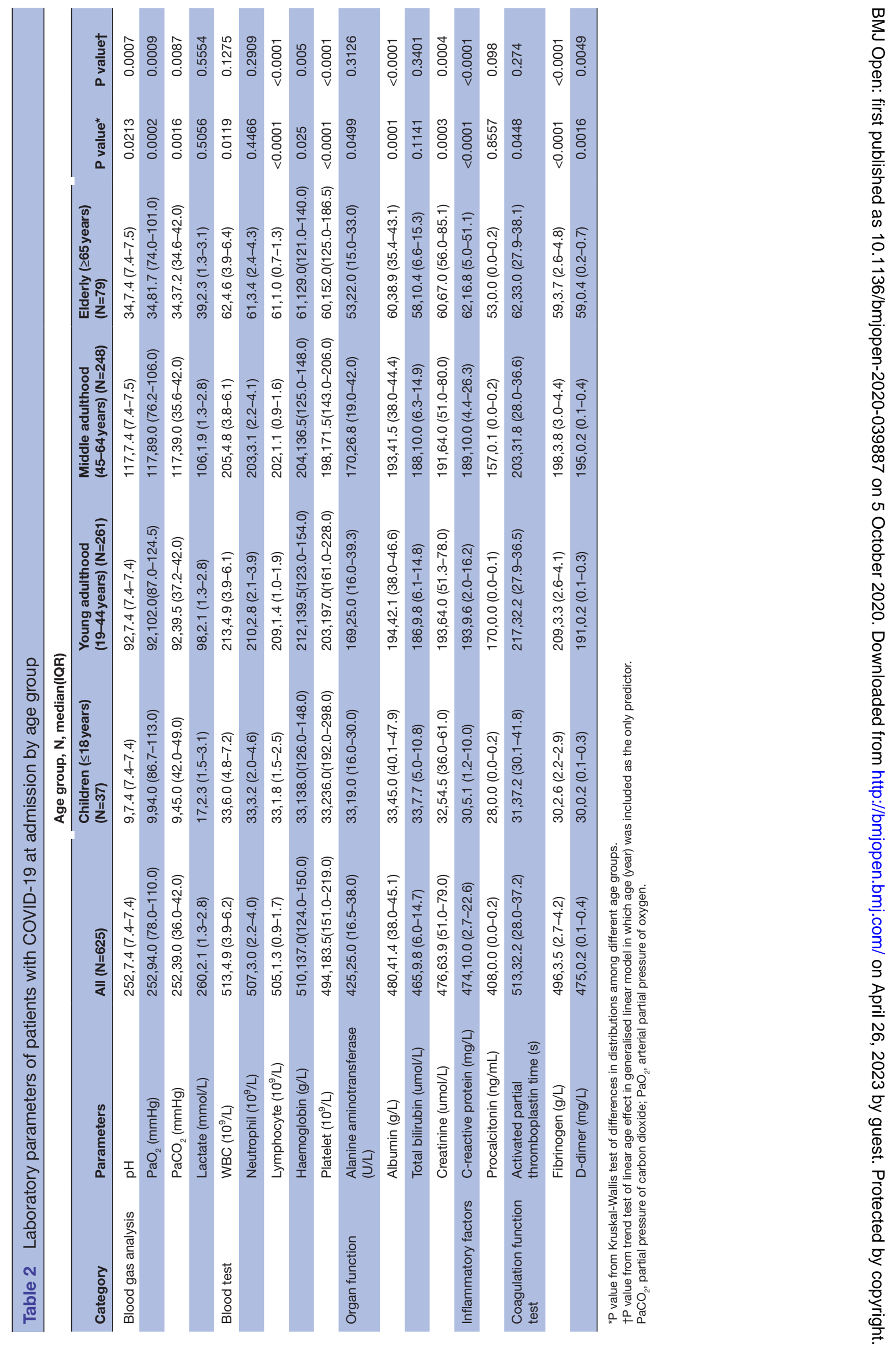


Table 3 Highest degree of disease severity of patients with COVID-19 during hospital stay by age group

\begin{tabular}{|c|c|c|c|c|c|c|}
\hline \multirow[b]{2}{*}{$\begin{array}{l}\text { Highest degree of } \\
\text { disease severity }\end{array}$} & \multirow[b]{2}{*}{ All (N=625) } & \multicolumn{4}{|c|}{ Age group, $n(\%)$} & \multirow[b]{2}{*}{$P$ value } \\
\hline & & $\begin{array}{l}\text { Children } \\
\text { ( } \leq 18 \text { years) } \\
(\mathrm{N}=37)\end{array}$ & $\begin{array}{l}\text { Young adulthood } \\
\text { (19-44 years) } \\
\text { ( } \mathrm{N}=261)\end{array}$ & $\begin{array}{l}\text { Middle adulthood } \\
\text { (45-64 years) } \\
(\mathrm{N}=248)\end{array}$ & $\begin{array}{l}\text { Elderly } \\
(\geq 65 \text { years) } \\
(\mathrm{N}=79)\end{array}$ & \\
\hline Asymptomatic & 24 (3.8\%) & 8 (21.6\%) & $5(1.9 \%)$ & $7(2.8 \%)$ & $4(5.1 \%)$ & $<0.0001$ \\
\hline Mild & 34 (5.4\%) & $11(29.7 \%)$ & 18 (6.9\%) & $4(1.6 \%)$ & $1(1.3 \%)$ & \\
\hline Moderate & $503(80.5 \%)$ & 18 (48.6\%) & 232 (88.9\%) & 205 (82.7\%) & 48 (60.8\%) & \\
\hline Severe & $30(4.8 \%)$ & $0(0.0 \%)$ & $4(1.5 \%)$ & $16(6.5 \%)$ & $10(12.7 \%)$ & \\
\hline Critically ill & 34 (5.4\%) & 0 (0.0\%) & $2(0.8 \%)$ & 16 (6.5\%) & 16 (20.3\%) & \\
\hline
\end{tabular}

gatherings. A previous study reported that COVID-19 in children was mainly caused by family transmission. ${ }^{11}$

Our study in Jiangsu, found that all patients were discharged with no deaths. Elderly patients were more than twice as likely to have a severe or critical illness compared with middle-aged patients, while a smaller proportion of young adult patients had severe symptoms, and child patients exhibited none. The ICU use and respiratory failure rate, and length of hospital stay increased with age. Many case studies have shown that older patients are refractory (not yielding to treatment or not significantly improved after treatment) and likely to be at higher risk of more severe disease including acute respiratory distress syndrome (ARDS), respiratory failure and death, ${ }^{12-19}$ while younger patients are more likely to have a mild or moderate type of COVID-19. ${ }^{112021}$ This is similar to SARS characteristics that, compared with adults and adolescents, the disease appears to be less severe in younger children. ${ }^{6}$

We found that initial symptoms including fever, cough and shortness of breath occurred more frequently among adults compared with child patients and especially shortness of breath was dramatically more common in elderly adult patients aged 65 years or older. This is consistent with the previous study showing that the older group ( $\geq 60$ years) had a higher rate of shortness of breath than the younger group $(<60$ years $) .{ }^{22}$ However, another study showed that only sore throat showed a significant difference between age groups but fever, cough, sputum and diarrhoea did not show differences, probably due to the small sample size. ${ }^{21}$ Another study which evaluated age difference only found four patients in total with symptoms of chest tightness or difficulty of breathing; however, the sample size (56 patients) was too small to draw any conclusions. ${ }^{8}$ Such symptoms may be the early signs of more severe illness and poorer outcomes in older patients. The frequency of these symptoms among adult patients in different age groups was similar except for shortness of breath, which was dramatically more common in elderly patients than younger adult groups in our study. This is different from the characteristics of influenza, where the initial clinical manifestations of frail elderly patients are usually subtle compared with young patients. ${ }^{23}{ }^{24}$ In our study, the difference in vital signs of
SBP and DBP on admission for different age groups were statistically significant (increasing with age) but may not be clinically significant.

Our study showed that the age differences in clinical outcomes may also have partially resulted from the increased incidence of comorbidities with age, including hypertension, coronary heart disease, chronic obstructive pulmonary disease and diabetes. This may have increased susceptibility to the virus infection; such comorbidities are identified as risk factors of more severe disease including respiratory failure and death in patients with COVID$19 .^{13}{ }^{17-19} 25$ Other explanations about why older people suffer poorer outcomes may be due to the higher prevalence of smoking and alcohol drinking in older patients with COVID-19 in Jiangsu. A history of smoking has been identified as a factor contributing to the progression of COVID-19 pneumonia. ${ }^{26}$

Our study demonstrated that the quadrant score and pulmonary opacity score increased with age, suggesting more severe abnormal imaging manifestation on admission among these older patients. This is consistent with the finding that the proportion of multiple lobe involvement in older patients was higher than in younger cases. ${ }^{8}$ Previous reports have also found some imaging differences by age groups; for example, primarily elderly patients were reported to have atypical imaging findings of consolidative opacities superimposed on ground-glass opacification, ${ }^{27}$ while paediatric patients showed more modest pulmonary involvement and less commonly reported consolidation complicated peripheral halo signs, compared with adults. ${ }^{28-30}$

Abnormal values in laboratory parameters in older patients may also be an early sign of, and a contribution to, severe illness and poor outcomes. This is consistent with previous studies showing the level of lymphocytes and albumin in the older cases was significantly lower and the level of C-reactive protein was higher than in the younger patients. ${ }^{81}$ Studies showed that albumin and C-reactive protein were associated with the progression of COVID-19 pneumonia, ${ }^{26}$ greater D-dimer on admission increased risk of in-hospital death, ${ }^{14}$ and organ and coagulation dysfunction (eg, higher D-dimer) contributed to the development of ARDS and progression from ARDS to death. ${ }^{16}$ 


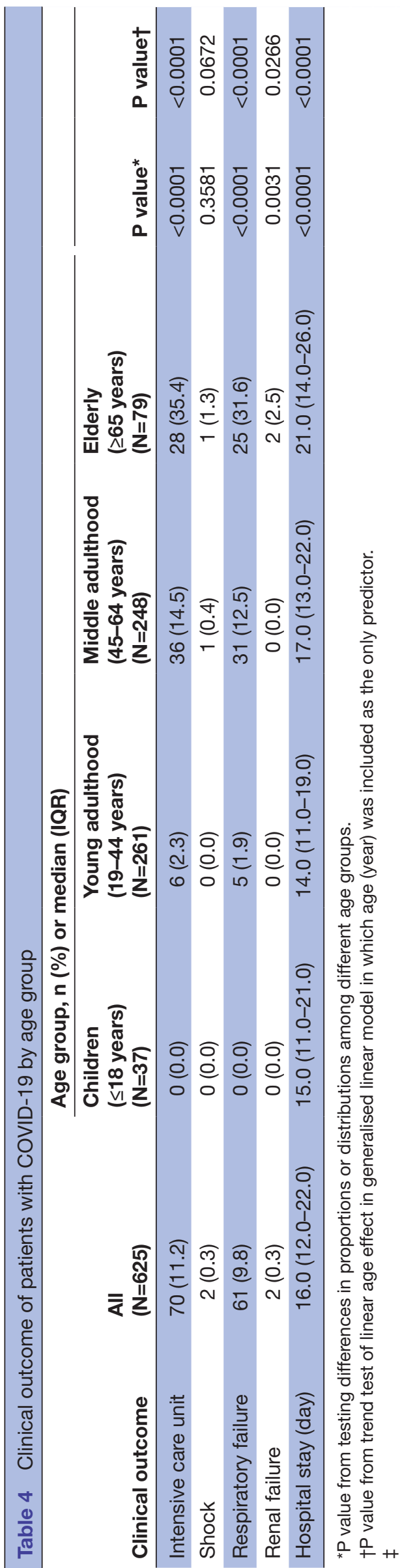

Our study indicated that lymphopenia (normal range: $\left.(0.3-3.0) \times 10^{9} / \mathrm{L}\right)$ was shown in all age groups, but white blood cell and lymphocytes counts were lower in older patients, indicating that live SARS-COV-2 virus stimulated poorer responses in older patients. The mechanism in old patients with a severe COVID-19 illness may be that older patients have a diminished immune response to the novel SARS-COV-2 virus, which is a defence mechanism against respiratory viruses and contributes to virus clearance. The thymic involution in older patients causes an age-related reduction of $\mathrm{T}$ cell repertoire diversity and defects in CD4+ and CD8+ T cell function and hence significantly reduces immune function (known as immunosenescence) ${ }^{31}{ }^{32}$ Immunosenescence makes many viral infections worse in older patients. ${ }^{33}$ But further research is necessary to investigate whether age differences in disease severity and outcomes of COVID-19 result from ageing of the immune system and reduced responsiveness. This is because some respiratory viruses could escape antiviral mechanisms and immune responses. ${ }^{34}$ For example, a study of H1N1pdm (respiratory viruses) on ferrets found no significant difference in viral clearance between young and adult subjects. ${ }^{35}$ However, studies found that the pulmonary pathology improved earlier in young ferrets, regulatory interleukin-10 (which is mainly produced by monocytes and lymphocytes) and interferon responses were more robust in young ferrets. ${ }^{35-37}$ Also, H1N1pdm infection triggered the formation of lung structures that resembled inducible bronchus-associated lymphoid tissues in young ferrets which contributes to pulmonary immune responses and were not seen in the adult ferrets with severe disease ${ }^{35-37}$ Some other studies demonstrated aged ferrets infected with influenza viruses had reduced antibody production and delayed peripheral blood T-cell responses compared with adult counterparts. ${ }^{31} 3238$ Another study reported that, except for immunosenescence in older people, age-related increases in levels of phospholipase could also result in a delay of immune response and poor outcomes after SARS-CoV infection. ${ }^{33}$ Overall, research focused on innate immunerelated mechanisms and viral clearance in patients with COVID-19 of different age groups may help determine the underlying mechanisms of disease severity.

Some other reasonable mechanisms of mild presentation in children include qualitatively different response to SARS-CoV-2, or different expression of ACE2 receptors required for SARS-CoV-2 infection, or different virusto-virus interaction and competition from other viruses limiting SARS-CoV-2 growth. ${ }^{10}$

A larger proportion of older patients in this study received supportive treatment and antiviral and antibiotic therapies which was due in part to the increasing proportion of severe or critical illness in older adults. Previous studies similarly showed that treatment was statistically different by age group. ${ }^{821}$

We believe that the findings from this study are generalisable to populations in similar settings (eg, outside the initial pandemic centre) for two reasons: (1) we 
included nearly all patients in Jiangsu province, China; (2) the study population consisted of cases confirmed by laboratory tests including those who were screened from suspected cases who had been to the pandemic centre (Wuhan), or had contact with people who had been to Wuhan or who had a confirmed diagnoses of COVID-19.

Our study has several limitations. First, the relatively short follow-up time and a very small proportion of patients who remained in hospital after the 14-day follow-up period may have resulted in incomplete estimates for disease severity. This would limit our interpretation of age differences in the burden associated with COVID-19. However, this impact is minor and may not strongly affect the study results because we included analyses of clinical outcomes at the end of the study. Second, we were unable to perform multiple regression analysis to control for possible bias in the observed age impact on clinical features and outcomes. As a result, the observed age differences may still be subject to possible confounding factors. Third, this is an observational and exploratory analysis in which many statistical tests have been performed. As a result, there may be some falsepositive results.

\section{CONCLUSIONS}

Older patients outside the pandemic centre of Wuhan, China, tended to have relatively more severe clinical infections and poorer clinical outcomes associated with COVID-19 compared with younger patients. Elderly patients aged 65 and older were at a much higher risk of developing severe or critical illness than other age groups. The ICU and respiratory failure rate, and length of hospital stay increased with age. Older patients had worse clinical outcomes, in part due to comorbidities in older people, and higher rates of smoking and drinking habits, and immune, organ and coagulation dysfunction on admission. In studying the pathogenesis and developing management strategies of COVID-19, age is confirmed as a critical factor in the severity of infection.

\section{Author affiliations}

${ }^{1}$ Department of Clinical Sciences, Liverpool School of Tropical Medicine, Liverpool, United Kingdom

${ }^{2}$ Jiangsu Provincial Key Laboratory of Critical Care Medicine, Department of Critical Care Medicine, Zhongda Hospital, School of Medicine, Southeast University, Nanjing, China

${ }^{3}$ Department of Radiology, Southeast University Zhongda Hospital, Nanjing, China

Contributors DW, SJ, YY and HL conceived and designed the study. HL, SL and YW contributed to the literature search. SL, YW, SJ, YY and DW contributed to data collection, quality checks and data management. DW, HL, SJ, SL, PP-H, YW and YY contributed to data analysis and results presentation. DW, HL, SL, SJ, PP-H and YY were responsible for results interpretation. HL, DW, PP-H, SL, YW, SJ, SL and YY contributed in the drafting and review of the manuscript.

Funding This work was supported, in part, by Research Grant 2020YFC0843700 67 from the Ministry of Science and Technology of the People's Republic of China.

Competing interests None declared.

Patient consent for publication Not required.
Ethics approval The study was approved by the Ethics Committee of Zhongda Hospital Affiliated to Southeast University (2020ZDSYLL013-P01 and 2020ZDSYLL019-P01).

Provenance and peer review Not commissioned; externally peer reviewed.

Data availability statement All data relevant to the study are included in the article or uploaded as supplemental information. All data are freely available within the appendices. No additional data available.

Open access This is an open access article distributed in accordance with the Creative Commons Attribution Non Commercial (CC BY-NC 4.0) license, which permits others to distribute, remix, adapt, build upon this work non-commercially, and license their derivative works on different terms, provided the original work is properly cited, appropriate credit is given, any changes made indicated, and the use is non-commercial. See: http://creativecommons.org/licenses/by-nc/4.0/.

\section{ORCID iDs}

Shenghong Ju http://orcid.org/0000-0002-2497-4286

Duolao Wang http://orcid.org/0000-0003-2788-2464

\section{REFERENCES}

1 Wang S, Guo L, Chen L, et al. A case report of neonatal COVID-19 infection in China. Clin Infect Dis 2020:ciaa225.

2 Sun D, Li H, XX L, et al. Clinical features of severe pediatric patients with coronavirus disease 2019 in Wuhan: a single center's observational study. World J Pediatr 2020.

3 Liu R, Han H, Liu F, et al. Positive rate of RT-PCR detection of SARSCoV-2 infection in 4880 cases from one hospital in Wuhan, China, from Jan to Feb 2020. Clin Chim Acta 2020;505:172-5.

4 Porcheddu R, Serra C, Kelvin D, et al. Similarity in case fatality rates (cfr) of COVID-19/SARS-COV-2 in Italy and China. J Infect Dev Ctries 2020;14:125-8.

5 Wong RSM, Wu A, To KF, et al. Haematological manifestations in patients with severe acute respiratory syndrome: retrospective analysis. BMJ 2003;326:1358-62.

6 Hon KLE, Leung CW, Cheng WTF, et al. Clinical presentations and outcome of severe acute respiratory syndrome in children. The Lancet 2003;361:1701-3.

7 Wang Y, Liu Y, Liu L, et al. Clinical outcome of 55 asymptomatic cases at the time of hospital admission infected with SARS-Coronavirus-2 in Shenzhen, China. $J$ Infect Dis 2020;221:jiaa119:1770-4.

8 Liu K, Chen Y, Lin R, et al. Clinical features of COVID-19 in elderly patients: a comparison with young and middle-aged patients. $J$ Infect 2020;80:e14-18.

9 National Health Commission, National Administration of Traditional Chinese Medicine. Diagnosis and treatment protocol for novel coronavirus pneumonia (trial version 7). Chin Med J 2020;133.

10 Brodin P. Why is COVID-19 so mild in children? Acta Paediatr 2020;109:apa.15271:1082-3.

11 Su L, Ma X, Yu H, et al. The different clinical characteristics of corona virus disease cases between children and their families in China - the character of children with COVID-19. Emerg Microbes Infect 2020;9:707-13.

12 Mo P, Xing Y, Xiao Y, et al. Clinical characteristics of refractory COVID-19 pneumonia in Wuhan, China. Clin Infect Dis 2020:ciaa270.

13 Yang X, Yu Y, Xu J, et al. Clinical course and outcomes of critically ill patients with SARS-CoV-2 pneumonia in Wuhan, China: a singlecentered, retrospective, observational study. Lancet Respir Med 2020;8:475-81.

14 Zhou F, Yu T, Du R, et al. Clinical course and risk factors for mortality of adult inpatients with COVID-19 in Wuhan, China: a retrospective cohort study. Lancet 2020;395:1054-62.

15 Shim E, Tariq A, Choi W, et al. Transmission potential and severity of COVID-19 in South Korea. Int $J$ Infect Dis 2020;93:339-44.

16 Wu C, Chen X, Cai Y, et al. Risk factors associated with acute respiratory distress syndrome and death in patients with coronavirus disease 2019 pneumonia in Wuhan, China. JAMA Intern Med 2020;180:934.

17 Liu K, Fang Y-Y, Deng Y, et al. Clinical characteristics of novel coronavirus cases in tertiary hospitals in Hubei Province. Chin Med $J$ 2020;133:1025-31.

18 Lai C-C, Liu YH, Wang C-Y, et al. Asymptomatic carrier state, acute respiratory disease, and pneumonia due to severe acute respiratory 
syndrome coronavirus 2 (SARS-CoV-2): facts and myths. J Microbiol Immunol Infect 2020;53:404-12.

19 Guo Y-R, Cao Q-D, Hong Z-S, et al. The origin, transmission and clinical therapies on coronavirus disease 2019 (COVID-19) outbreak an update on the status. Military Med Res 2020;7:11.

20 Qiu H, Wu J, Hong L, et al. Clinical and epidemiological features of 36 children with coronavirus disease 2019 (COVID-19) in Zhejiang, China: an observational cohort study. Lancet Infect Dis 2020;S14733099:30198-5.

21 Liu Y, Mao B, Liang S, et al. Association between age and clinical characteristics and outcomes of COVID-19. Eur Respir J 2020;55:2001112.

22 Lian J, Jin X, Hao S, et al. Analysis of epidemiological and clinical features in older patients with corona virus disease 2019 (COVID-19) out of Wuhan. Clin Infect Dis 2020:ciaa242.

23 Pop-Vicas A, Gravenstein S. Influenza in the elderly: a mini-review. Gerontology 2011;57:397-404.

24 Wong PL, Sii HL, P'ng CK, et al. The effects of age on clinical characteristics, hospitalization and mortality of patients with influenza-related illness at a tertiary care centre in Malaysia. Influenza Other Respi Viruses 2020;14:286-93.

25 Leung C. Clinical features of deaths in the novel coronavirus epidemic in China. Rev Med Virol 2020;30:e2103.

26 Liu W, Tao Z-W, Wang L, et al. Analysis of factors associated with disease outcomes in hospitalized patients with 2019 novel coronavirus disease. Chin Med J 2020;133:1032-8.

27 Salehi S, Abedi A, Balakrishnan S, et al. COVID-19: a systematic review of imaging findings in 919 patients. Am J Roentgenol 2019:1-7.

28 Xia W, Shao J, Guo Y, et al. Clinical and CT features in pediatric patients with COVID-19 infection: different points from adults. Pediatr Pulmonol 2020;55:1169-74.
29 Liu H, Liu F, Li J, et al. Clinical and CT imaging features of the COVID-19 pneumonia: focus on pregnant women and children. $J$ Infect 2020;80:30118-3.

$30 \mathrm{Li} \mathrm{W}$, Cui $\mathrm{H}$, Li K, et al. Chest computed tomography in children with COVID-19 respiratory infection. Pediatr Radiol 2020.

31 Maue AC, Yager EJ, Swain SL, et al. T-Cell immunosenescence: lessons learned from mouse models of aging. Trends Immunol 2009;30:301-5

32 Yager EJ, Ahmed M, Lanzer K, et al. Age-Associated decline in T cell repertoire diversity leads to holes in the repertoire and impaired immunity to influenza virus. J Exp Med 2008;205:711-23.

33 Vijay R, Hua X, Meyerholz DK, et al. Critical role of phospholipase A2 group IID in age-related susceptibility to severe acute respiratory syndrome-CoV infection. J Exp Med 2015;212:1851-68.

34 Vareille M, Kieninger E, Edwards MR, et al. The airway epithelium: soldier in the fight against respiratory viruses. Clin Microbiol Rev 2011;24:210-29.

35 Huang SSH, Banner D, Degousee N, et al. Differential pathological and immune responses in newly weaned ferrets are associated with a mild clinical outcome of pandemic $2009 \mathrm{H} 1 \mathrm{~N} 1$ infection. J Virol 2012;86:13187-201.

36 Hwang JY, Randall TD, Silva-Sanchez A. Inducible BronchusAssociated lymphoid tissue: taming inflammation in the lung. Front Immunol 2016;7:258.

37 Randall TD. Bronchus-associated lymphoid tissue (BALT) structure and function. Adv Immunol 2010;107:187-241.

38 Paquette SG, Huang SSH, Banner D, et al. Impaired heterologous immunity in aged ferrets during sequential influenza A H1N 1 infection. Virology 2014;464-465:177-83. 